\title{
Setosphaeria rostrata
}

National Cancer Institute

\section{Source}

National Cancer Institute. Setosphaeria rostrata. NCI Thesaurus. Code C127747.

A species of fungi in the phylum Ascomycota characterized by mature conidia with a distinct protruding hilum. This species is commonly found in soils, but is an opportunistic agent of sinusitis, keratitis, and CNS vasculitis as well as cutaneous and subcutaneous mycoses. 